\title{
Preliminary study of contrast-enhanced ultrasound in combination with blue dye vs. indocyanine green fluorescence, in combination with blue dye for sentinel lymph node biopsy in breast cancer
}

Yidong Zhou ${ }^{1 *+}$, Yan Li ${ }^{1+}$, Feng Mao ${ }^{1}$, Jing Zhang ${ }^{2}$, Qingli Zhu ${ }^{2}$, Songjie Shen ${ }^{1}$, Yan Lin ${ }^{1}$, Xiaohui Zhang ${ }^{1}$, He Liu ${ }^{2}$, Mengsu Xiao ${ }^{2}$ Y Yuxin Jiang ${ }^{2}$ and Qiang Sun ${ }^{1 *}$

\begin{abstract}
Background: This preliminary study aimed to examine the feasibility of sentinel lymph node biopsy (SLNB) using contrast-enhanced ultrasound (CEUS) vs. indocyanine green fluorescence (ICG), combined with blue dye in patients with breast cancer.

Methods: This was a retrospective study of consecutive female patients with invasive stage I-III (based on preoperative physical examination and imaging) primary breast cancer at the Peking Union Medical College Hospital between 01/2013 and 01/2015 who underwent preoperative SLNB by ICG + blue dye or CEUS + blue dye. The numbers of detected SLNs, detection rates, and recurrence-free survival (RFS) rates were compared between the two groups.
\end{abstract}

Results: A total of 443 patients were included. The detection rates of SLNs in the CEUS + blue dye and ICG + blue dye groups were 98.4 and $98.1 \%$, respectively $(P=0.814)$. The average numbers of SLNs detected per patient showed no significant difference between the two groups (3.06 \pm 1.33 and $3.12 \pm 1.31$ in the CEUS + blue dye and ICG + blue dye groups, respectively; $P=0.659$ ). After a median follow-up of 46 months, five patients in the CEUS + blue dye group and 15 in the ICG + blue dye group had recurrence. RFS rates showed no significant difference $(P=0.55)$.

Conclusion: This preliminary study suggests that CEUS + blue dye and ICG + blue dye are both feasible for SLN detection in breast cancer.

Keywords: Breast cancer, Sentinel lymph node, Biopsy, Contrast-enhanced ultrasound, Indocyanine green fluorescence

\section{Background}

Breast cancer is currently the most common malignancy in Chinese women $[1,2]$. Recent years have witnessed an increase in the incidence of early breast cancer because of related screening programs, improved women's breast cancer awareness, and ameliorated imaging technologies.

\footnotetext{
* Correspondence: zhouydpumch@126.com; sunqiangpumch@126.com ${ }^{\dagger}$ Yidong Zhou and Yan Li contributed equally to this work.

'Department of Breast Surgery, Peking Union Medical College Hospital, Peking Union Medical College, Chinese Academy of Medical Sciences, Beijing 100730, People's Republic of China

Full list of author information is available at the end of the article
}

Invasive breast cancer is of particular significance because of its propensity to spread to local lymph nodes and then to other organs/sites. Axillary lymph nodes are the most common sites of regional metastasis, and sentinel lymph node (SLN) biopsy (SLNB) is necessary for tumor staging and prognosis. Axillary lymph node dissection (ALND) allows the sampling of lymph nodes but is associated with significant morbidities such as upper extremity numbness, infection, and lymphedema [3]. SLNB allows the first step of staging, and ALND can be omitted in patients with negative SLNs, reducing the likelihood of complications [3]. SLNs are defined as the

(c) The Author(s). 2019 Open Access This article is distributed under the terms of the Creative Commons Attribution 4.0 International License (http://creativecommons.org/licenses/by/4.0/), which permits unrestricted use, distribution, and 
initial lymph nodes that drain the breast; thus, their histological condition is considered to represent that of the entire axillary region [4].

The current standard SLNB method involves the injection of a technetium-labeled nanocolloid and blue dye interstitially into the breast, either around the tumor via the periareolar procedure [5]. Use of a radioisotope combined with blue dye is a common method for SLNB, but its shortcomings are not negligible [6]. First, SLNs cannot be detected until many hours have elapsed after radioactive colloid injection, which is a challenge to schedule management. Secondly, patients and healthcare workers may express reluctance to radiation exposure [5]. Thirdly, access to radioisotopes is restricted in some countries. These factors limit the use of SLNB worldwide, especially in hospitals of less developed regions.

In China, using blue dye alone is common in SLNB. Although SLNs are dyed, blue dye cannot indicate their localization prior to skin incision. As a result, the identification rate is not as high as that of the dual method (radiotracer and blue dye) [7, 8]. Therefore, alternative techniques for SLNB are actively sought. Such methods should yield a satisfactory SLN identification rate and avoid the need for radioisotopes.

Therefore, new techniques are being developed for SLNB. Among them, indocyanine green fluorescence (ICG) and contrast-enhanced ultrasound (CEUS) have some advantages [5, 6, 9-21]. Recent studies confirmed that ICG or CEUS alone is feasible and safe for SLNB. However, there are limited data on the benefits of combining ICG or CEUS with blue dye.

Therefore, the aim of the present preliminary study was to examine the effectiveness of SLN identification using CEUS vs. ICG, in combination with blue dye. In addition, we attempted to compare breast cancer recurrence rates between both techniques. The present results provide a proof-of-concept for designing prospective trials.

\section{Methods}

\section{Ethics statement}

This study was approved by the independent ethical committee/institutional review board of Peking Union Medical College Hospital (PUMCH). We obtained permission from PUMCH to collect data from the Breast Surgery Department Database. As this was a retrospective study of anonymized data without any contact with the patients, individual consent was not required. The study was performed in accordance with the relevant guidelines and regulations.

\section{Patients}

A retrospective review of the Breast Surgery Department database of PUMCH was performed. Consecutive female patients aged $\geq 18$ years, with invasive primary breast cancer (stages I-III; based on pre-operative physical examination and imaging), who underwent preoperative SLNB using ICG or CEUS combined with blue dye between January 2013 and January 2015 were included for analysis. Preoperatively, these patients had no clinical (as examined by palpation) or radiological signs of lymph node invasion. Patients who received neoadjuvant systemic therapy (including chemotherapy and endocrine therapy) were excluded, as well as those with bilateral breast cancer or a history of axillary surgery. All eligible patients in the database had complete medical information. No patient was lost to follow-up. Follow-up was censored on January 19, 2018.

\section{Operative procedures}

The choice of the SLNB procedure was based on the surgeon's experience and preference at the time of surgery. All SLNB procedures were performed by the same team of senior and skilled breast surgeons. Undiluted methylene blue (Bailunsi Co., Tianjin, China, $10 \mathrm{mg} / \mathrm{ml}$ ) was used for both SLNB procedures.

For ICG + blue dye, ICG (Dandongyichuang Co., Liaoning, China, 25-mg vial) was first dissolved in $5.0 \mathrm{ml}$ sterile water $(5.0 \mathrm{mg} / \mathrm{ml}$ stock solution $)$. Then, $1.25 \mathrm{ml}$ of the stock solution was diluted in $5.0 \mathrm{ml}$ sterile water for injection $(1.0 \mathrm{mg} / \mathrm{ml})$. Before surgery, $0.2 \mathrm{ml}$ of methylene blue $(10 \mathrm{mg} / \mathrm{ml})$ and $0.2 \mathrm{ml}$ of ICG $(1.0 \mathrm{mg} / \mathrm{ml})$ were injected intradermally into the periareolar region. The breast was gently massaged for $5 \mathrm{~min}$. Next, the lights were turned off and a photodynamic eye (PDE) camera (Hamamatsu Photonics Co., Hamamatsu, Japan) was used to trace the lymphatic flow. The location of the skin incision for the SLNB was selected as the point where the fluorescent signal disappeared (Fig. 1a). After dissection, the camera was used to confirm the fluorescent signals of SLNs. Blue, fluorescent, and palpable suspicious nodes were all removed and assessed (Fig. 1b).

For CEUS + blue dye, CEUS localization of SLNs was performed in the ultrasound room about $30 \mathrm{~min}$ before surgery. Ultrasound was performed on an Acuson S2000 (Siemens Medical Systems, Erlangen, Germany) with 18L6HD and 9L4 high-frequency linear array probes, using contrast pulse sequences (CPS). Low mechanical index $(\mathrm{MI})$ values were used $(\mathrm{MI}=0.06)$ to reduce cavitation and microbubble destruction. Sonovue (Bracco Imaging, Milan, Italy) was used as the contrast agent. The Sonovue powder was mixed with $2.0 \mathrm{ml}$ of sterile saline. The ultrasound contrast agent $(0.4 \mathrm{ml})$ was injected intradermally into the periareolar area, and the injection area was gently massaged for 10-30 s. Subcutaneous lymphatic channels could be visualized immediately on CPS. Enhanced lymph nodes were detected by moving the probe along the channels (Fig. 1c). Grey 


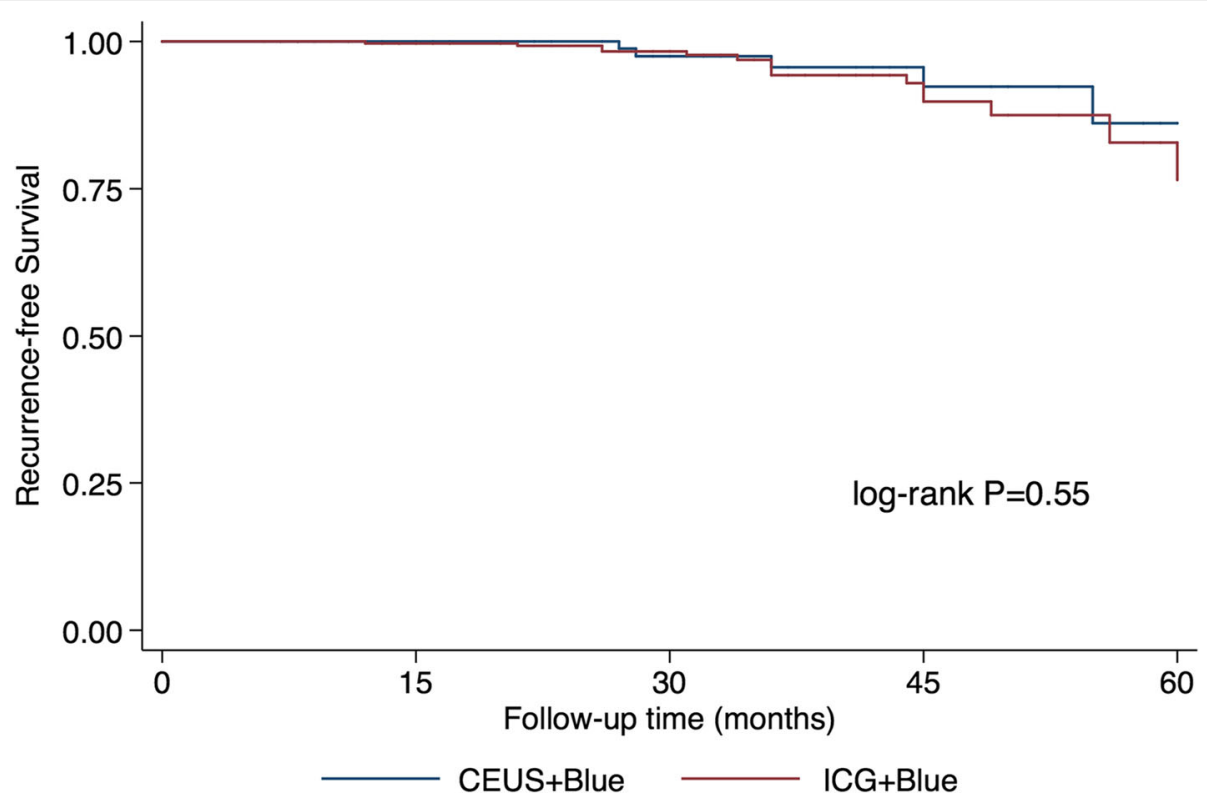

Fig. 1 Sentinel lymph node (SLN) localization images. a Fluorescent signal mapping of the lymphatic flow and SLNs. b SLN detection by the indocyanine green (ICG) + blue dye method. c Subcutaneous lymphatic channels and SLNs detected by contrast enhanced ultrasound (CEUS)

scale or live dual images were used to confirm the presence of SLNs. Once identified, lymphatic duct and SLNs were marked on the skin to guide the incision. After CEUS localization, the patients were transferred to the operating room (OR), where the blue dye tracing procedure was performed as described above. Blue, CEUSlocalized, and palpable suspicious nodes were removed and assessed.

\section{Pathological analysis}

All the harvested SLNs underwent routine histopathological examination at approximately 2 -mm intervals. Immunohistochemistry was performed for the confirmation of suspected metastases. All analyses were performed by the same team of pathologists.

\section{Data collection}

As this was a retrospective study, the patients were grouped according to the SLNB procedure received. Tumor characteristics and demographic information were collected from medical records, including age, menopausal status, tumor size, tumor grade, tumor stage, ER, PR, HER2, and detailed information about SLNB procedures. Follow-up data were reviewed from the hospital's followup system. The adverse events routinely documented after SLNB included lymphedema, infection, sensory deficit, and shoulder function deficit.

\section{Statistical analysis}

The detection rate of SLNs was defined as the number of patients with SLNs identified by the labeling technique divided by the total number of patients administered the technique. Categorical data were compared by the twotailed chi-square test. Quantitative data were compared by Student's t-test. Recurrence-free survival (RFS) was estimated by the Kaplan-Meier method. A two-sided log-rank test for time-to-event endpoint was used. Differences were considered statistically significant at $P<0.05$. Statistical analyses were performed with STATA (version 14.0, StataCorp LP, College Station, TX, USA).

\section{Results}

Patient and tumor characteristics

Between January 2013 and January 2015, a total of 443 patients were operated and included in this study. The ICG + blue dye technique was used in 316 (71.3\%) individuals, and CEUS + blue dye in 127 (28.7\%) patients. Table 1 presents the characteristics of both groups. There were no significant differences in age, menopausal status, and breast surgical treatment between the two groups (all $P>$ 0.05). There were also no significant differences between the two groups in tumor size, tumor grade, stage, lymphovascular invasion, estrogen receptor (ER), progesterone receptor (PR), and human epidermal growth factor receptor 2 (HER2) (all $P>0.05$ ). No adverse reactions or complications related to the ICG procedure, microbubbles, or blue dye injection were recorded.

\section{Assessment of the two novel dual techniques}

Among the 127 patients in the CEUS + blue dye group, SLN detection was successful in 125 (98.4\%). Of the 316 patients administered ICG + blue dye, SLN detection 
Table 1 Characteristics of the patients

\begin{tabular}{|c|c|c|c|}
\hline Characteristics & CEUS + blue dye $(n=127)$ & ICG + blue dye $(n=316)$ & $P$ \\
\hline Age, years & & & 0.891 \\
\hline Mean \pm SD & $45.0 \pm 14.5$ & $46.9 \pm 15.0$ & \\
\hline Menopausal status, n (\%) & & & 0.987 \\
\hline Premenopausal & $72(56.7)$ & $180(57.0)$ & \\
\hline Postmenopausal & $47(37.0)$ & $115(36.4)$ & \\
\hline Unknown & $8(6.3)$ & $21(6.6)$ & \\
\hline Tumor stage, $n(\%)$ & & & 0.287 \\
\hline $\mathrm{T} 1$ & $80(63.0)$ & $217(68.7)$ & \\
\hline $\mathrm{T} 2$ & $40(31.5)$ & $90(28.5)$ & \\
\hline T3 & $7(5.5)$ & $9(2.8)$ & \\
\hline Tumor grade, $n(\%)$ & & & 0.466 \\
\hline G1 & $20(15.7)$ & $54(17.1)$ & \\
\hline G2 & $64(50.4)$ & $139(44.0)$ & \\
\hline G3 & $43(33.9)$ & $123(38.9)$ & \\
\hline Tumor stage, $n(\%)$ & & & 0.841 \\
\hline I & $74(58.3)$ & $193(61.1)$ & \\
\hline$\|$ & $50(39.4)$ & $117(37.0)$ & \\
\hline III & $3(2.3)$ & $6(1.9)$ & \\
\hline LVI, n (\%) & & & 0.745 \\
\hline Yes & $7(5.5)$ & $20(6.3)$ & \\
\hline No & $120(94.5)$ & $296(93.7)$ & \\
\hline ER status, $n(\%)$ & & & 0.682 \\
\hline Positive & $97(76.4)$ & $230(72.8)$ & \\
\hline Negative & $23(18.1)$ & $69(21.8)$ & \\
\hline unknown & $7(5.5)$ & $17(5.4)$ & \\
\hline PR status, $n(\%)$ & & & 0.768 \\
\hline Positive & $91(71.7)$ & $219(69.4)$ & \\
\hline Negative & $29(22.8)$ & $82(25.9)$ & \\
\hline unknown & $7(5.5)$ & $15(4.7)$ & \\
\hline HER2 status, $n(\%)$ & & & 0.879 \\
\hline Positive & $24(18.9)$ & $56(17.7)$ & \\
\hline Negative & $95(74.8)$ & $243(76.9)$ & \\
\hline Equivocal /unknown & $8(6.3)$ & $17(5.4)$ & \\
\hline Breast surgery, n (\%) & & & 0.518 \\
\hline Lumpectomy & $50(39.4)$ & $135(42.7)$ & \\
\hline Mastectomy & 77 (60.6) & $181(57.3)$ & \\
\hline
\end{tabular}

CEUS Contrast-enhanced ultrasound, ICG Indocyanine green, SD Standard deviation, LVI Lymphovascular invasion, ER Estrogen receptor, PR Progesterone receptor, HER2 Human epidermal growth factor receptor 2

was successful in 310 (98.1\%). The SLN detection rate showed no significant difference $(P=0.814)$. The numbers of SLNs identified showed no significant difference between the two groups $(3.06 \pm 1.33$ and $3.12 \pm 1.31$, respectively; $P=0.659$ ). There were no significant differences in the positive SLN rate between the two groups.
Precisely, there were 13 (10.2\%) patients (11 with macrometastases, 1 with micrometastasis, and 1 with isolated tumor cells) with positive SLNs in the CEUS + blue dye group, and 36 (11.4\%) (30 with macrometastases, 3 with only micrometastases, and 3 with isolated tumor cells) in the ICG + blue dye group $(P=0.726)$ (Table 2$)$. 
Table 2 Comparison of sentinel lymph node biopsy results between the two groups

\begin{tabular}{llll}
\hline & CEUS + blue dye $(n=127)$ & ICG + blue dye $(n=316)$ & $P$ \\
\hline Identification rate of SLNs, $n(\%)$ & $125 / 127(98.4)$ & $310 / 316(98.1)$ & 3.814 \\
Number of SLNs identified per patient, mean \pm SD & $3.06 \pm 1.33$ & $3.12 \pm 1.31$ & $36 / 316(11.4)$ \\
SLN metastasis, $n(\%)$ & $13 / 127(10.2)$ & $12.10 \pm 3.21$ & 0.659 \\
Time consumption of SLN localization in the OR (min) & $11.01 \pm 3.56$ & 0.726 \\
\hline
\end{tabular}

CEUS Contrast-enhanced ultrasound, ICG Indocyanine green, SLN Sentinel lymph node, SD Standard deviation, OR Operating room

The time to SLN localization in the OR showed no significant difference between the two groups (11.01 \pm 3.56 vs. $12.10 \pm 3.21 \mathrm{~min}, P=0.105$ ) (Table 2 ).

All the 49 SLN-positive patients underwent complete ALND, except 1 (isolated tumor cells) in the CEUS + blue dye group, and 5 (including 2 and 3 with micrometastases and isolated tumor cells, respectively) in the ICG + blue dye group.

\section{Recurrence-free survival}

Median follow-up was 46 (range, 8-60) months. Among the 443 patients, 20 (4.5\%) had tumor recurrence. Five (3.9\%) individuals in the CEUS + blue dye group had recurrence, including 1, 2 and 2 with axillary recurrence, ipsilateral breast/chest wall recurrence and bone metastasis, respectively. A total of 15 (4.7\%) patients in the ICG + blue dye group showed recurrence, including 3, 4, 4 and 4 with axillary recurrence, ipsilateral breast/chest wall recurrence, bone metastasis and lung metastasis, respectively. The 3year RFS was $95.6 \%$ in the CEUS + blue dye group versus $94.3 \%$ in the ICG + blue dye group $(P=0.55)$ (Fig. 2). No patient died during follow-up.

Regarding cases with axillary recurrence, the patient in the CEUS + blue dye group was a 35-year old woman, whose SLNB showed $0 / 3$ positive SLN. In the $\mathrm{ICH}+$ blue dye group, 2 patients (51- and 42-year old women) had 0/3 and 0/4 positive SLN, respectively; the third patient, a 38year old woman, had $1 / 4$ positive SLN for a macrometastasis and underwent ALND, with $0 / 18$ positive lymph node.

\section{Discussion}

ICG, a novel technique for SLNB, is increasingly used in clinical practice. The SLNB detection rate with ICG alone ranges from 93.1 to $100 \%$, for $1.5-5.4$ sentinel lymph nodes sampled per patient $[5,6,11,16-18,20,21]$. The combined use of the conventional blue dye with ICG fluorescence could improve SLN localization and potentially reduce surgical time [22-24]. This combination makes the SLNB procedure easier to perform.

CEUS is another new technique for SLNB and has been validated in a pig melanoma model $[25,26]$. Subsequent studies confirmed that CEUS is safe and reliable for SLNB. In 2010, Sever et al. used CEUS for SLNB, and reported a sensitivity of up to $89 \%$ [19]. Cox et al. reported a study of 347 breast cancer patients and revealed a detection rate of $87.7 \%$ [14]. Esfehani et al. detected lymphatic pathways and SLNs by CEUS alone, with a sensitivity as high as 96\% [15]. The CEUS enhancement patterns may help recognize metastatic SLNs and determine the total axillary nodal burden [12, 13]. In addition, CEUS and ICG allow real-time observation of the lymphatic flow in the axilla. Therefore, CEUS can help surgeons plan surgery prior to any incision [13].

In the present preliminary study, the detection rates of SLNs for the two techniques were high and comparable: 98.4\% for CEUS + blue dye, and 98.1\% for ICG + blue dye. These rates are similar to that (96\%) reported in the literature $[27,28]$. The two techniques detected $>3$ SLNs per patient, without a significant difference. Regarding time consumption in the OR, because the CEUS procedure was performed outside the OR, it is reasonable to expect a shorter localization time in the OR for the CEUS + blue dye technique, implying that the latter method might have a potentially higher efficiency of OR usage. However, no significant difference was observed in the present study between the two methods.

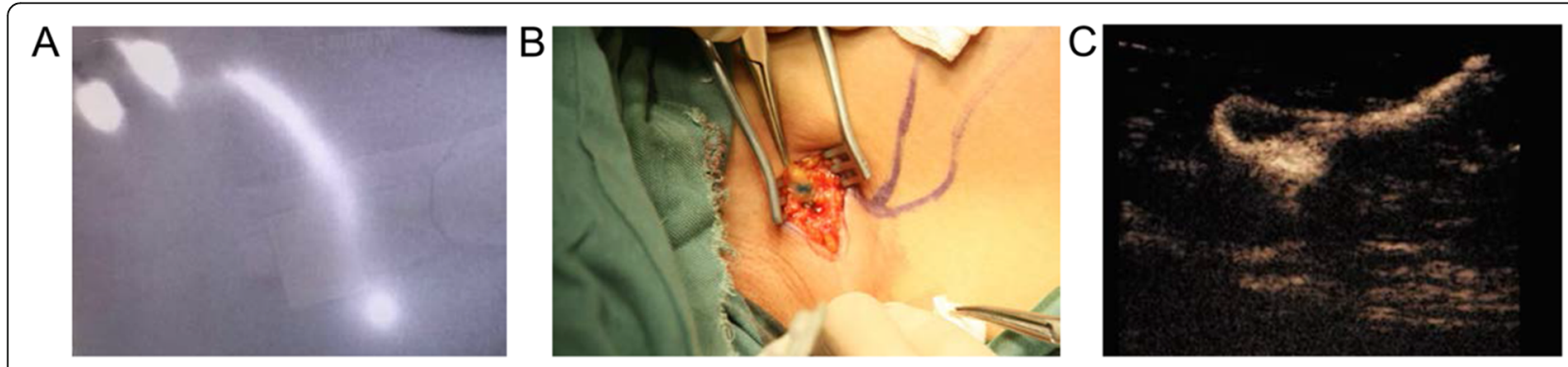

Fig. 2 Recurrence-free survival in the contrast enhanced ultrasound (CEUS) + blue dye and indocyanine green (ICG) + blue dye groups 
Without performing ALND in all patients, the real falsenegative rate could not be determined, but the rate of ipsilateral axillary recurrence could be used as an imperfect adjunct. In the present study, the recurrence rates in the ipsilateral axilla were low [1/127 (0.8\%) and 3/316 (1.0\%)], suggesting that false-negative rates for both approaches were most likely low. The reported false-negative rate for SLNB is $5-13 \%$, depending upon the number of SLNs sampled, the SLNB method applied, and the cancer type $[27,29,30]$. In the present study, false negative rates based on regional recurrence were lower than previously reported, suggesting a probable underestimation. Among the four patients with axillary recurrence, only one had a positive SLN; she underwent ALND, and all the dissected lymph nodes were negative. Indeed, it is still possible to miss positive lymph nodes during ALND, or the surgeon may decide to not dissect all three levels. In addition, lymph nodes harboring isolated tumor cells may remain clinically negative for a long time and even never develop overt metastasis [31], although conflicting data were reported [32]. Nevertheless, a meta-analysis revealed that dual techniques for SLNB result in lower false negative rates than the use of blue dye alone [28].

In addition to axillary recurrence cases, six (1.4\%) patients had ipsilateral breast/chest wall recurrence and 10 (2.3\%) developed distant metastasis during the 46-month follow-up. These rates were similar to those reported previously [33-35]. However, such comparison should be interpreted with caution because rates may vary widely when considering the type of breast cancer, the HER2 status, surgical and adjuvant treatments, ethnicity, life style habits, and the follow-up itself.

This study had limitations. ALND was not performed in all patients, and the false-negative rates of the two novel techniques could not be evaluated. Even though there were no significant differences in baseline patient and tumor characteristics between the two groups, a retrospective analysis inevitably has some biases, e.g. we were limited to the data available in medical charts. Furthermore, the surgeons were free to select the preferred method for different patients, and the exact reasons for method selection were usually not indicated in patient charts. Finally, patients assessed by the radiotracer + blue dye technique could not be included because our center does not use radiotracers.

\section{Conclusion}

Overall, the present preliminary study suggested that CEUS + blue dye and ICG + blue dye are both feasible techniques for SLNB in breast cancer. Randomized controlled trials including the radiotracer + blue dye gold standard technique are required to confirm the feasibility, efficacy, and safety of these two novel techniques before their introduction into mainstream clinical practice.

\section{Abbreviations}

CEUS: Contrast enhance ultrasound; CPS: Contrast pulse sequences;

ER: Estrogen receptor; HER2: Human epidermal growth factor receptor 2; ICG: Indocyanine green fluorescence; MI: Mechanical index; OR: Operating room; PDE: Photodynamic eye; PR: Progesterone receptor; PUMCH: Peking Union Medical College Hospital; RFS: Recurrence-free survival; SLNB: Sentinel lymph nodes biopsy

\section{Acknowledgements}

We thank Dr. Xi Cao and Dr. Jialin Zhao for enlightening advices.

\section{Authors' contributions}

YDZ and YL conceived, designed and coordinated the study, performed the experiments, analyzed the data, and wrote the manuscript. FM, JZ, QLZ, SJS, YL, XHZ, HL, MSX, YXJ and QS carried out data collection, data analysis, and manuscript revision. All authors reviewed the data and approved the final version of the manuscript.

\section{Funding}

This work was supported by the Beijing Natural Science Foundation (\#7172168), the National Key R\&D Program of China (\#2016YFC1302601), and the Beijing Municipal Science and Technology Key Development Program (\#D161100000816005). The funders had no role in study design, data collection and analysis, decision to publish, or manuscript preparation.

\section{Availability of data and materials}

The raw data are available upon request to the corresponding author and/or to the first author

\section{Ethics approval and consent to participate}

This study was approved by the independent ethical committee/institutional review board of Peking Union Medical College Hospital (PUMCH). We obtained permission from PUMCH to collect data from the Breast Surgery Department Database. For this type of retrospective study, formal consent was not required. The study was performed in accordance with the relevant guidelines and regulations.

\section{Consent for publication}

Not applicable.

\section{Competing interests}

The authors declare that they have no competing interests.

\section{Author details}

${ }^{1}$ Department of Breast Surgery, Peking Union Medical College Hospital, Peking Union Medical College, Chinese Academy of Medical Sciences, Beijing 100730, People's Republic of China. ²Department of Ultrasound, Peking Union Medical College Hospital, Peking Union Medical College, Chinese Academy of Medical Sciences, Beijing 100730, People's Republic of China.

Received: 24 May 2019 Accepted: 16 September 2019

Published online: 11 October 2019

\section{References}

1. Chen W, Zheng R, Baade PD, Zhang S, Zeng H, Bray F, Jemal A, Yu XQ, He J. Cancer statistics in China, 2015. CA Cancer J Clin. 2016;66(2):115-32.

2. Fan L, Strasser-Weippl K, Li JJ, St Louis J, Finkelstein DM, Yu KD, Chen WQ, Shao ZM, Goss PE. Breast cancer in China. Lancet Oncol. 2014;15(7):e279-89.

3. Veronesi U, Paganelli G, Viale G, Luini A, Zurrida S, Galimberti V, Intra M, Veronesi $P$, Robertson $C$, Maisonneuve $P$, et al. A randomized comparison of sentinel-node biopsy with routine axillary dissection in breast cancer. $\mathrm{N}$ Engl J Med. 2003:349(6):546-53.

4. Morton DL, Wen DR, Wong JH, Economou JS, Cagle LA, Storm FK, Foshag LJ, Cochran AJ. Technical details of intraoperative lymphatic mapping for early stage melanoma. Arch Surg. 1992;127(4):392-9.

5. Ahmed M, Purushotham AD, Douek M. Novel techniques for sentinel lymph node biopsy in breast cancer: a systematic review. Lancet Oncol. 2014;15(8):e351-62.

6. Benson J. Indocyanine green fluorescence for sentinel lymph node detection in early breast Cancer. Ann Surg Oncol. 2016;23(1):6-8. 
7. Sugie T, Sawada T, Tagaya N, Kinoshita T, Yamagami K, Suwa H, Ikeda T, Yoshimura K, Niimi M, Shimizu A, et al. Comparison of the indocyanine green fluorescence and blue dye methods in detection of sentinel lymph nodes in early-stage breast cancer. Ann Surg Oncol. 2013;20(7):2213-8.

8. Hung WK, Chan CM, Ying M, Chong SF, Mak KL, Yip AW. Randomized clinical trial comparing blue dye with combined dye and isotope for sentinel lymph node biopsy in breast cancer. Br J Surg. 2005;92(12):1494-7.

9. Aoyama K, Kamio T, Ohchi T, Nishizawa M, Kameoka S. Sentinel lymph node biopsy for breast cancer patients using fluorescence navigation with indocyanine green. World J Surg Oncol. 2011;9:157.

10. Cui $X$, Ignee A, Nielsen MB, Schreiber-Dietrich D, De Molo C, Pirri C, Jedrzejczyk M, Christoph DF. Contrast enhanced ultrasound of sentinel lymph nodes. J Ultrason. 2013;13(52):73-81.

11. Pitsinis V, Provenzano E, Kaklamanis L, Wishart GC, Benson JR. Indocyanine green fluorescence mapping for sentinel lymph node biopsy in early breast cancer. Surg Oncol. 2015;24(4):375-9.

12. Xie F, Zhang D, Cheng L, Yu L, Yang L, Tong F, Liu H, Wang S, Wang S. Intradermal microbubbles and contrast-enhanced ultrasound (CEUS) is a feasible approach for sentinel lymph node identification in early-stage breast cancer. World J Surg Oncol. 2015;13:319.

13. Zhao J, Zhang J, Zhu QL, Jiang YX, Sun Q, Zhou YD, Wang MQ, Meng ZL, Mao XX. The value of contrast-enhanced ultrasound for sentinel lymph node identification and characterisation in pre-operative breast cancer patients: a prospective study. Eur Radiol. 2018;28(4):1654-61.

14. Cox K, Sever A, Jones S, Weeks J, Mills P, Devalia H, Fish D, Jones P. Validation of a technique using microbubbles and contrast enhanced ultrasound (CEUS) to biopsy sentinel lymph nodes (SLN) in pre-operative breast cancer patients with a normal grey-scale axillary ultrasound. Eur J Surg Oncol. 2013;39(7):760-5.

15. Esfehani MH, Yazdankhah-Kenari A, Omranipour R, Mahmoudzadeh HA Shahriaran S, Zafarghandi MR, Amoli HA. Validation of contrast enhanced ultrasound technique to wire localization of sentinel lymph node in patients with early breast Cancer. Indian J Surg Oncol. 2015;6(4):370-3.

16. Guo J, Yang H, Wang S, Cao Y, Liu M, Xie F, Liu P, Zhou B, Tong F, Cheng L, et al. Comparison of sentinel lymph node biopsy guided by indocyanine green, blue dye, and their combination in breast cancer patients: a prospective cohort study. World J Surg Oncol. 2017;15(1):196.

17. Hokimoto N, Sugimoto T, Namikawa T, Funakoshi T, Oki T, Ogawa M, Fukuhara H, Inoue K, Sato T, Hanazaki K. A novel color fluorescence navigation system for intraoperative transcutaneous lymphatic mapping and resection of sentinel lymph nodes in breast cancer: comparison with the combination of gamma probe scanning and visible dye methods. Oncology. 2018;94(2):99-106.

18. Liu J, Huang L, Wang N, Chen P. Indocyanine green detects sentinel lymph nodes in early breast cancer. J Int Med Res. 2017:45(2):514-24.

19. Sever AR, Mills P, Jones SE, Cox K, Weeks J, Fish D, Jones PA. Preoperative sentinel node identification with ultrasound using microbubbles in patients with breast cancer. AJR Am J Roentgenol. 2011;196(2):251-6.

20. Sugie T, Ikeda T, Kawaguchi A, Shimizu A, Toi M. Sentinel lymph node biopsy using indocyanine green fluorescence in early-stage breast cancer: a meta-analysis. Int J Clin Oncol. 2017;22(1):11-7.

21. Shen S, Xu Q, Zhou Y, Mao F, Guan J, Sun Q. Comparison of sentinel lymph node biopsy guided by blue dye with or without indocyanine green in early breast cancer. J Surg Oncol. 2018;117(8):1841-7.

22. Hirche C, Murawa D, Mohr Z, Kneif S, Hunerbein M. ICG fluorescence-guided sentinel node biopsy for axillary nodal staging in breast cancer. Breast Cancer Res Treat. 2010;121(2):373-8.

23. van der Vorst JR, Schaafsma BE, Verbeek FP, Hutteman M, Mieog JS, Lowik CW, Liefers GJ, Frangioni JV, van de Velde CJ, Vahrmeijer AL. Randomized comparison of near-infrared fluorescence imaging using indocyanine green and 99(m) technetium with or without patent blue for the sentinel lymph node procedure in breast cancer patients. Ann Surg Oncol. 2012;19(13):4104-11.

24. Hojo T, Nagao T, Kikuyama M, Akashi S, Kinoshita T. Evaluation of sentinel node biopsy by combined fluorescent and dye method and lymph flow for breast cancer. Breast. 2010;19(3):210-3.

25. Goldberg BB, Merton DA, Liu JB, Thakur M, Murphy GF, Needleman L, Tornes A, Forsberg F. Sentinel lymph nodes in a swine model with melanoma: contrast-enhanced lymphatic US. Radiology. 2004;230(3):727-34.

26. Mattrey RF, Kono Y, Baker K, Peterson T. Sentinel lymph node imaging with microbubble ultrasound contrast material. Acad Radiol. 2002; 9(Suppl 1):S231-5.
27. Kataria K, Srivastava A, Qaiser D. What is a false negative sentinel node biopsy: definition, reasons and ways to minimize it? Indian J Surg. 2016; 78(5):396-401.

28. Pesek S, Ashikaga T, Krag LE, Krag D. The false-negative rate of sentinel node biopsy in patients with breast cancer: a meta-analysis. World J Surg. 2012;36(9):2239-51.

29. Lee SA, Lee HM, Lee HW, Yang BS, Park JT, Ahn SG, Jeong J, Kim SI. Risk factors for a false-negative result of sentinel node biopsy in patients with clinically node-negative breast Cancer. Cancer Res Treat. 2018;50(3):625-33.

30. Li J, Chen X, Qi M, Li Y. Sentinel lymph node biopsy mapped with methylene blue dye alone in patients with breast cancer: a systematic review and meta-analysis. PLoS One. 2018;13(9):e0204364.

31. Degnim AC, Zakaria S, Boughey JC, Sookhan N, Reynolds C, Donohue JH, Farley DR, Grant CS, Hoskin T. Axillary recurrence in breast cancer patients with isolated tumor cells in the sentinel lymph node [AJCC NO(i+)]. Ann Surg Oncol. 2010;17(10):2685-9.

32. Pepels MJ, de Boer $M$, Bult $P$, van Dijck JA, van Deurzen $\mathrm{CH}_{\text {, Menke- }}$ Pluymers MB, van Diest PJ, Borm GF, Tjan-Heijnen VC. Regional recurrence in breast cancer patients with sentinel node micrometastases and isolated tumor cells. Ann Surg. 2012;255(1):116-21.

33. Cao JQ, Olson RA, Tyldesley SK. Comparison of recurrence and survival rates after breast-conserving therapy and mastectomy in young women with breast cancer. Curr Oncol. 2013;20(6):e593-601.

34. Saad ED, Squifflet P, Burzykowski T, Quinaux E, Delaloge $S$, Mavroudis D, Perez E, Piccart-Gebhart M, Schneider BP, Slamon D, et al. Disease-free survival as a surrogate for overall survival in patients with HER2-positive, early breast cancer in trials of adjuvant trastuzumab for up to 1 year: a systematic review and meta-analysis. Lancet Oncol. 2019;20(3):361-70.

35. O'Rorke MA, Murray LJ, Brand JS, Bhoo-Pathy N. The value of adjuvant radiotherapy on survival and recurrence in triple-negative breast cancer: a systematic review and meta-analysis of 5507 patients. Cancer Treat Rev. 2016;47:12-21.

\section{Publisher's Note}

Springer Nature remains neutral with regard to jurisdictional claims in published maps and institutional affiliations.

Ready to submit your research? Choose BMC and benefit from:

- fast, convenient online submission

- thorough peer review by experienced researchers in your field

- rapid publication on acceptance

- support for research data, including large and complex data types

- gold Open Access which fosters wider collaboration and increased citations

- maximum visibility for your research: over $100 \mathrm{M}$ website views per year

At BMC, research is always in progress.

Learn more biomedcentral.com/submissions 